\title{
Acceso y uso de las Tecnologías de la información y las Comunicaciones (TICs) en el aprendizaje. El Caso de los Jóvenes Preuniversitarios en Caldas, Colombia
}

\author{
Carlos E. Marulanda(1), Jaime Giraldo(2) y Marcelo López ${ }^{(1)}$ \\ (1) Universidad Nacional de Colombia, Facultad de Administración, Departamento de Administración. \\ Campus Palogrande. Calle 27 No. 64-60, Manizales-Colombia y Universidad de Caldas, Facultad de \\ Ingeniería, Departamento de Sistemas e Informática, Calle 65 No. 26-10, Manizales-Colombia. (e-mail: \\ cemarulandae@unal.edu.co, carlose@ucaldas,edu.co,mlopez@ucaldas.edu.co,mlopezt@unal.edu.co). \\ (2) Universidad Nacional de Colombia, Facultad de Ingeniería, Departamento de Ingeniería Industrial, \\ campus La nubia Manizales-Colombia. (e-mail: jaiagiraldog@unal.du.co)
}

Recibido May. 30, 2014; Aceptado Jul. 10, 2014; Versión final recibida Jul. 28, 2014

\section{Resumen}

Se estudia el uso y apropiación de las tecnologías de información y comunicaciones (TICs) por parte de jóvenes de último año de bachillerato (preuniversitarios), en colegios públicos y privados de diversos municipios del Departamento de Caldas en Colombia. Se utilizaron métodos de observación, análisis y síntesis y un tipo de estudio descriptivo, exploratorio y correlacional. Se destaca que el acceso y uso se está generando desde la internet, el teléfono móvil, la facilidad de acceso y el interés por las tecnologías, pero no se están aprovechando adecuadamente dichas tecnologías en asuntos de aprendizaje, Este estudio aporta información a la comunidad académica para definir una línea base que permita potenciar la apropiación de las tecnologías en procesos de aprendizaje.

\section{Access and use of Information and Communication Technologies (ICTs) in the learning process The Case of Pre- University Students in Caldas, Colombia}

\begin{abstract}
The use and appropriation of information and communication technologies (ICTs) by young high school seniors (pre-university students) in public and private schools in several municipalities Department of Caldas, Colombia is studied. For this purpose, methods of observation, analysis and synthesis and a type of descriptive, exploratory, correlational study were used. It is noted that the access and use is being generated from the internet, mobile phones, ease access and interest in technology, but technologies are not properly use in matters related to learning. This study provides the academic community information for defining a baseline to promote appropriation of technologies in learning processes.
\end{abstract}




\section{INTRODUCCIÓN}

A la sociedad de la información se le ha designado como un nuevo tipo de sociedad que se la ha generado por el efecto re-configurador de las posibilidades abiertas por las tecnologías de la información y comunicación (TICs). Castells (2006) cuando se refiere a la sociedad de la información precisa que es una forma específica de organización social en la que la generación, el procesamiento y la transmisión de información se convierten en las fuentes fundamentales de la productividad y la colaboración; estas nuevas condiciones tecnológicas surgen en esta época histórica de la humanidad, como una relación mediada por factores institucionales y por factores organizativos y de recursos humanos adecuados a un sistema nuevo de producción y de gestión.

Las TICs y sus actuales mediaciones (redes sociales, videojuegos, teléfonos inteligentes, televisión digital y servicios web) están generando en los jóvenes nuevas formas de informarse, interactuar, divertirse y aprender (Bernete, 2009), además que es innegable que la generación de estudiantes que se está educando hoy ha crecido de la mano del computador y por tanto se ha habituado al uso de juegos, programas y acceso a la red y estas nuevas costumbres podrían facilitar el aprendizaje soportado por computadora (Giraldo, Toro, \& Jaramillo, 2013), por lo que es posible que los futuros universitarios lleguen al alma máter con habilidades en manejo de información, bien definidas. Existen trabajos previos de uso de las TICs para la educación no centrados en apropiación y en aprendizaje como (Ofsted, 2002), (Boschma, 2008), (Piscitelli, 2009), (López et al., 2011), (Muñoz, Córdova y Priego, 2012),(Osuna y Luna, 2011) y (Quinche y González, 2011), orientados principalmente a expresar como es el acceso y uso de herramientas TICs para la educación.

La mejor tecnología no es en muchos casos la más avanzada, sino la que mejor se adapte a las necesidades específicas de un medio en donde ejerce su función, a veces es preferible usar y apropiar acorde a los requerimientos de los jóvenes, en vez de generar productos inutilizados con mejor tecnología. La acción tecnológica como tal es neutra y no crea las comunidades, crea una tecnología como base de estas comunidades e incide en las capacidades personales, grupales y organizacionales, en la forma en que los jóvenes interactúan en comunidad (Benavides, 1998). Es claro que la tecnología por sí sola no implica una buena formación, pero sin duda, es casi imposible conseguir una buena educación sin tecnología. El uso de TICs considera instrumentos de integración de contenidos, así como herramientas para el proceso de enseñanza aprendizaje, generando situaciones susceptibles de ser sometidas al análisis, evaluación e investigación permanente (Enrique y Alzugaray, 2013)

Como colectivo definido, los jóvenes establecen relaciones informales para compartir tareas y responsabilidades en un contexto común, estas acciones con la mediación de las TICs pueden tipificarse como grupos colaborativos entre los jóvenes: grupos con intereses especiales, centros y comunidades de competencia (CoC) y comunidades de práctica como lo plantea (O'Sullivan y Azeem, 2007). Estas estructuras se pueden potenciar para que los jóvenes gestionen tiempo, manejen líneas de autoridad y liderazgo, distribuyan el poder, focalicen actividades con visiones y metas y manejo de la cooperación comunes. El acceso y uso de las TICs para el aprendizaje induce a pensar un ambiente de aprendizaje como sujeto que actúa con el ser humano y lo transforma en un espacio y un tiempo en movimiento, donde los participantes desarrollan capacidades, competencias, habilidades y valores. Los ambientes de aprendizaje incluyen currículo, tiempo, mediaciones y entornos físicos y/o virtuales (Alcaldia Mayor de Bogota, 2013). En la sociedad del aprendizaje, el aprendizaje es visto como productor de conocimiento: científico, tecnológico, intelectual, práctico, tradicional, legal, colectivo, del sentido común y del saber; del conocimiento de los procesos relacionales de comunicación y lenguaje que se dan al interior de una cultura. $\mathrm{O}$ el Know what (saber qué), know why (saber por qué) en lecturas de libros, bases de datos y otros; know how (saber cómo), know who (saber quién) en experiencia práctica, relaciones maestro-aprendiz y práctica cotidiana entre otros. Es todo lo asociado al aprendizaje permanente, como actividad de formación, realizada de manera continua, y que tiene por objeto mejorar las cualificaciones, los conocimientos y las capacidades de las personas (Comunidades Europeas, 2007).

Existen ambientes de inter - aprendizaje donde aprender es una relación social entre las personas para potenciarse y enriquecerse; el meta - aprendizaje se realiza cuando se va más allá del aprendizaje y la persona conoce sus procesos y modos de aprender, lo que le posibilita el poder incidir, dirigir y mejorar la calidad del aprendizaje. El intra - aprendizaje se da cuando la persona se interioriza y accede a sus propios sentimientos, potenciando sus pensamientos e inteligencia (Moreno, 2010). Todos estos ambientes están mediados por las TICs y la posibilidad de interconectividad para posibilitar conocimientos, experiencias y acceso a medios; el aprendizaje usando y apropiándose de las TICs se refiere a la transformación de conocimientos y comportamientos o desempeños a través de la experiencia y las capacidades, todo esto posibilitado por las tecnologías digitales de estos tiempos (Lonsdale et al., 2010). 
Como lo señala OCDE (2010), "los jóvenes se encuentran en plena experimentación de nuevas formas de socialización y de adquisición de capital social a las que las TICs están contribuyendo en gran medida". Para la OCDE las competencias de las TICs para los jóvenes están asociadas a: i) Habilidades funcionales TICs, que incluyen habilidades relevantes para un buen uso de las diferentes aplicaciones; ii) Habilidades TICs para aprender, que incluyen habilidades que combinan las actividades cognitivas y de orden superior con habilidades funcionales para el uso y manejo de estas aplicaciones; y iii) Habilidades propias del siglo $\mathrm{XXI}$, necesarias para la sociedad del conocimiento donde el uso de las TICs es una condición necesaria.

Aún más, cuando los jóvenes como nativos digitales, nacieron en la era digital y son usuarios permanentes de las TICs con una habilidad consumada, son tecnófilos, dado que con las tecnologías digitales satisfacen sus necesidades de entretenimiento, socialización, diversión, comunicación, interacción e información y como reto de formación (García et al., 2006). Según estos autores, estos nuevos usuarios enfocan su vida, el aprendizaje y los juegos de nuevas formas, porque captan rápidamente la información multimedia de imágenes y videos, por encima de la de texto; porque son capaces de acceder a datos simultáneamente de múltiples fuentes y esperan respuestas inmediatas.

En el mundo de hoy se aborda la exclusión y la diversidad desde la base de la igualdad de oportunidades, poniéndose un énfasis especial en el diseño cosmopolita, la accesibilidad y la supresión de obstáculos como instrumento de inserción, integración, inclusión y regulación. Bajo este enfoque, el acceso a la sociedad del conocimiento es una condición básica en el proceso de equivalencia de oportunidades (UNESCO, 2005). Desde esta enfoque, es preciso que el diseño y la aplicación de las tecnologías no se oriente como diseño especializado o adaptado a la limitación, sino guiado y pensado para las necesidades de las personas, bajo entornos diferentes, de unos colectivos a otros. La alta versatilidad y riqueza de campo en el ámbito de las TICs y la inclusión, es un nuevo reto al que se debe hacer frente a las múltiples demandas que se plantean y por las numerosas desigualdades que se pudieran producir de no regular y coordinar debidamente su aprovechamiento (Cuesta \& López, 2008).

Pero aparece la brecha digital, la cual ha sido definida como "la distancia 'tecnológica' entre individuos, familias, empresas, grupos de interés, países y áreas geográficas en sus oportunidades en el acceso a la información y a las tecnologías de la comunicación y en el uso de internet para un amplio rango de actividades" (ALADI, 2003). Las TICs pueden ser un elemento clave para mejorar calidad de vida y, en algunos momentos, una de las pocas opciones para poder acceder a un mejor currículum escolar, u ofrecer su integración social y laboral. En definitiva, para normalizar sus condiciones de vida. Pero también pueden ser usadas con fines meramente lúdicos y hedónicos o incluso delictivos (Braslavsky, 2004).

En el Departamento de Caldas (Colombia) se han realizado importantes acercamientos a los temas de ciencia, tecnología e innovación que ponen de manifiesto la necesidad de avanzar en ellos, promover estrategias de promoción de empresas innovadoras y buscar alianzas entre las diferentes instituciones, en pro del desarrollo de Caldas, tal como se evidencia el proyecto de investigación doctoral titulado: modelo de evaluación de gestión del conocimiento en el sector TI del triángulo del café de Colombia.

Específicamente Tamayo y López (2013) proponen que deben desplegarse estrategias, líneas de investigación y acciones de apropiación de las TICs para los jóvenes del departamento, en la perspectiva del entorno y contexto de territorio y de priorizar el uso y apropiación de las ellas para la ciencia y la tecnología. Por lo tanto esta propuesta tiene como objetivo aportar al diseño de una agenda de ciencia y tecnología para Caldas con proyección hacia el triángulo del café (región de Colombia, compuesta por los departamentos de Caldas, Risaralda y Armenia y que se caracteriza por tener la mayor producción del café del País), que contribuya al fortalecimiento del sistema regional de innovación y al desarrollo de la región a partir del aprovechamiento endógeno de los recursos.

Esta propuesta tiene como objetivo aportar al diseño de una agenda de ciencia y tecnología para Caldas con proyección hacia el triángulo del café, que contribuya al fortalecimiento del sistema regional de innovación y al desarrollo de la región a partir del aprovechamiento endógeno de los recursos. Para los jóvenes busca un acercamiento prospectivo que pretende articular el desarrollo científico tecnológico de Caldas con el panorama que plantean los próximos veinte años del siglo XXI, siendo conscientes de que el mundo ha cambiado radicalmente en los últimos años y que megatendencias de mucha fuerza como la globalización, la sociedad del conocimiento y la tecnología y auge de la información vienen con fuerza en el nuevo milenio. La educación es fundamental en el desarrollo de la ciencia y la tecnología, ello implica trabajar en los diferentes niveles básico, secundario y superior, pero es necesario abordar además de los temas de infraestructura, los componentes de investigación y desarrollo (lo pedagógico como concepto de educación no asistencial sino de investigación), de manera que se logren innovaciones organizacionales determinantes de la competitividad (Tamayo y López, 2013). 
En contraste, la organización para la cooperación y el desarrollo económicos (OCDE), reveló los resultados de la pruebas internacionales Pisa 2012, realizadas a más de 510.000 escolares y respaldadas por las más importantes instituciones de evaluación educativa en el mundo, las cuales se aplican cada tres años a jóvenes de 15 años, de 65 países, para evaluar el desempeño de los estudiantes en las áreas de: lectura, matemáticas y ciencias y Colombia ocupa el puesto 62 (con 376 puntos), convirtiéndose en la segunda peor puntuación de Latinoamérica, solo superada por Perú con el puesto 65 (368 puntos) (Espectador, 2000), lo que ratifica aún más apropiar los resultados de esta investigación.

\section{MATERIALES Y MÉTODOS}

Para el desarrollo se hace una investigación exploratoria, para analizar e identificar las principales variables del modelo de evaluación, una investigación descriptiva, para conocer las situaciones predominantes con la representación exacta de los procesos, y una investigación de tipo correlacional para encontrar la relación entre las variables. El tratamiento estadístico implica realizar un análisis de correlación, considerando valores significativos cercanos a 1 y un análisis de componentes principales considerando los primeros cuatro factores.

\section{Determinación de la muestra}

Se aplicó la encuesta de manera personal a una muestra de la población de 995 estudiantes de secundaria del grado 10 y 11 (preuniversitarios) de colegios públicos y privados de los municipios de Manizales, Villamaría, Neira, Chinchiná, La Dorada y Norcasia, pertenecientes a las regiones centro-sur y oriente del departamento de Caldas. Previamente a la valoración, se desarrolló una prueba piloto de la encuesta con expertos sobre el tema, además de una revisión con algunos de los actores académicos. Para la estructuración del cuestionario se formularon preguntas en escala Likert, las cuales se calificaron de 1 a 5 , donde 1 nunca, 2 ocasionalmente, 3 a veces, 4 habitualmente y 5 siempre.

\section{Variables evaluadas}

Las variables se fundamentan en los planteamientos desarrollados por (Bernete, 2009), considerando las interrelaciones y las perspectivas dinámicas de la educación y las competencias en TICs, por medio de métricas, herramientas e indicadores específicos, ligados a las competencias individuales. Estas se agruparon tal como se observa en la Tabla 1. Para revisión de los resultados de la encuesta, revisar en las Internet 1 (2014).

Tabla 1.Variables evaluadas

\begin{tabular}{|l|l|}
\hline Variable & Indicador \\
\hline Acceso a computador & Desde la casa, el colegio, la casa de los amigos, café internet y relacionados (Acom) \\
\hline Acceso a internet & Desde la casa, el colegio, la casa de los amigos, café internet y relacionados (Aint) \\
\hline Acceso a telefonía móvil & Desde un smartphone, 1G o 2G (Atem) \\
\hline Acceso a videoconsola & Desde la casa, el colegio, la casa de los amigos, café internet y relacionados (Avdj) \\
\hline Frecuencia de uso en la casa & $\begin{array}{l}\text { Del televisor, computador, teléfono celular, internet, videoconsola de juegos, juegos } \\
\text { por computador, redes sociales y servicios web educativos (Fcas) }\end{array}$ \\
\hline $\begin{array}{l}\text { Frecuencia de uso en el } \\
\text { colegio }\end{array}$ & $\begin{array}{l}\text { Del televisor, computador, teléfono celular, internet, videoconsola de juegos, juegos } \\
\text { por computador, redes sociales y servicios web educativos (Fcol) }\end{array}$ \\
\hline Uso del computador e internet & $\begin{array}{l}\text { Para escribir (hacer trabajos académicos), escuchar y descargar mús videos y } \\
\text { películas, jugar, tratamiento de imágenes, buscar información concreta, usar redes } \\
\text { sociales, usar correo electrónico, cursos virtuales y otros (Upcin) }\end{array}$ \\
\hline Uso del teléfono móvil & $\begin{array}{l}\text { Para tener conversaciones con amigos, hablar con familiares, intercambio de } \\
\text { mensajes, conectarse a internet, jugar y hacer fotos (Utem) }\end{array}$ \\
\hline Horas de uso en semana & Del televisor, computador, internet, teléfono móvil y videoconsola de juegos (Hsem) \\
\hline $\begin{array}{l}\text { Horas de uso en fin de } \\
\text { semana }\end{array}$ & Del televisor, computador, internet, teléfono móvil y videoconsola de juegos (Hfin) \\
\hline $\begin{array}{l}\text { Impresiones al usar el } \\
\text { computador }\end{array}$ & Para diversión, aprovechar el tiempo libre, compañía y aprender (lupc) \\
\hline Impresiones al usar el Internet & Para diversión, aprovechar el tiempo libre, compañía y aprender (luin) \\
\hline $\begin{array}{l}\text { Impresiones al usar } \\
\text { videojuegos }\end{array}$ & Para: diversión, aprovechar el tiempo libre, compañía y aprender (luvdj) \\
\hline Interés por las tecnologías & $\begin{array}{l}\text { Como televisor, computador personal, internet, videoconsola, teléfonos móviles y } \\
\text { tableta (Inter) }\end{array}$ \\
\hline $\begin{array}{l}\text { Facilidad para vivir sin la } \\
\text { siguiente tecnología }\end{array}$ & Televisor, computador, internet, teléfono móvil y videoconsola de juegos (Fac) \\
\hline
\end{tabular}




\section{RESULTADOS Y DISCUSIÓN}

El proceso de recolección de datos se hizo personalmente con los estudiantes de los grados 10 y 11 de los colegios públicos y privados en el momento del registro, para asegurarse que tuvieran comprensión del tema, además de hacer un muestreo aleatorio de comprobación. Se presenta a su vez la matriz de correlación, ver Tabla 2, la cual indica un alto grado de correlación entre las variables evaluadas, lo que hace posible aplicar el método de componentes principales. En la variable uso computador e internet, los resultados se graficaron tal como se observa en la Figura 1.

Tabla 2.Matriz de correlación (valor $p$ ) entre variables

\begin{tabular}{|c|c|c|c|c|c|c|c|c|c|c|c|c|c|c|}
\hline \multicolumn{15}{|c|}{ Variables } \\
\hline \multicolumn{2}{|l|}{ Acom } & & & & & & & & & & & & & \\
\hline Aint & ,002 & & & & & & & & & & & & & \\
\hline Atem & ,003 & 0 & & & & & & & & & & & & \\
\hline Avdj & ,038 & ,318 & ,211 & & & & & & & & & & & \\
\hline Fcas & ,012 & ,001 & ,002 & ,258 & & & & & & & & & & \\
\hline Fcol & ,535 & ,038 & ,864 & ,028 & ,418 & & & & & & & & & \\
\hline Upcin & ,203 & ,035 & ,708 & ,021 & ,736 & ,012 & & & & & & & & \\
\hline Utem & ,872 & ,326 & ,409 & ,455 & ,021 & ,001 & ,084 & & & & & & & \\
\hline Hcem & ,003 & ,021 & ,218 & ,012 &, 587 & ,037 & ,039 & ,021 & & & & & & \\
\hline Hfin & ,732 & ,345 & ,342 & ,227 & ,405 & ,304 & ,474 & ,734 & ,021 & & & & & \\
\hline lupc & ,025 & ,004 & ,025 & ,003 & ,002 & ,006 & ,037 & ,035 & ,018 & ,673 & & & & \\
\hline Iuin & ,034 & ,039 & ,219 &, 556 & 013 & ,483 & ,276 & ,508 & ,725 & ,013 & 011 & & & \\
\hline luvdj & ,183 & ,042 & ,012 & ,028 & ,022 & ,034 & ,012 & ,001 & ,033 & ,586 & ,767 & ,821 & & \\
\hline Inter & ,036 & ,733 & ,650 & ,781 & ,438 & ,009 & ,588 & ,037 & ,052 & ,018 & ,018 & ,874 & ,584 & \\
\hline Fac & ,026 & ,019 & ,023 & ,015 & ,587 & ,032 & ,021 & ,575 & ,011 & 171 & ,006 & ,012 & ,787 & ,047 \\
\hline
\end{tabular}

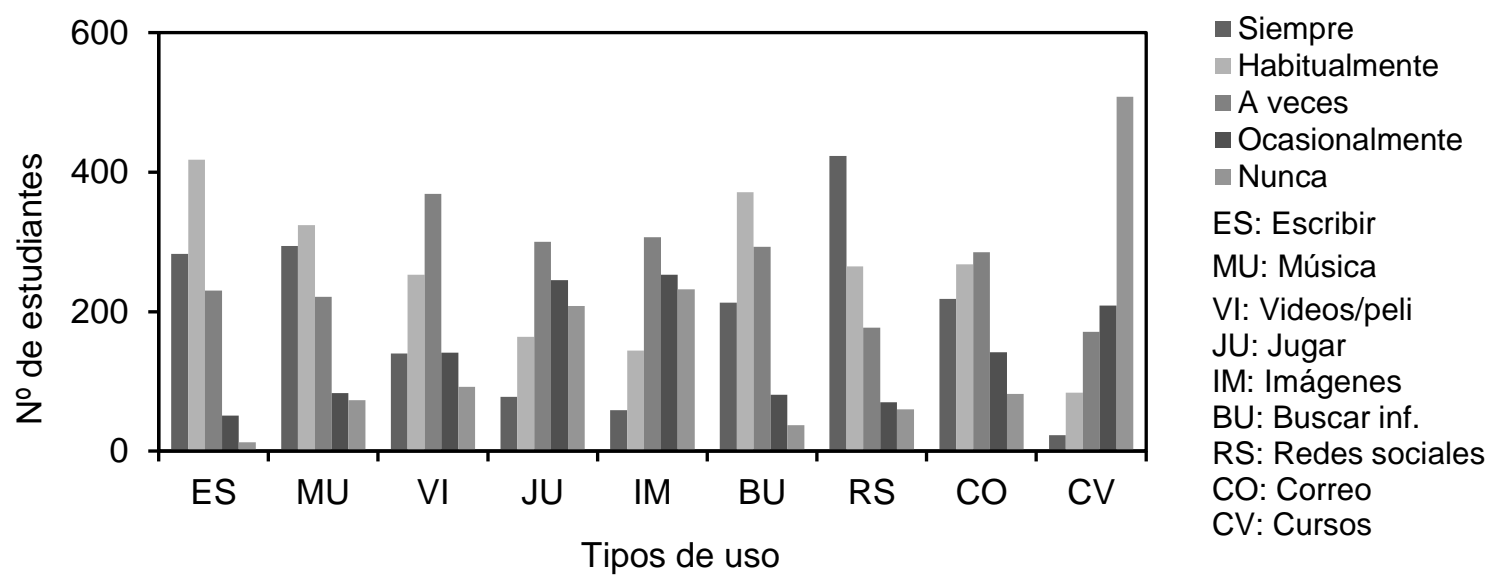

Fig. 1: Frecuencia y uso de computador e internet de los estudiantes de colegio de 10 y 11

De acuerdo a la escala de calificación, un $42 \%$ de los estudiantes de 10 y 11 , siempre utilizan el computador y el internet, en manejo de redes sociales, otro $42 \%$, para escribir sus trabajos académicos, un $37 \%$ para buscar información y en contraste un $51 \%$, nunca los utilizan para estudiar cursos virtuales. Cifras relativamente bajas para el aprovechamiento real de su potencial en cuanto a lo académico, lo que se pudiera explicar por la falta de acceso, dada la ausencia de recursos para adquirirlas, considerando el nivel de pobreza de los municipios, o quizás por la falta de capacitación, asesoría y acompañamiento de padres e instituciones en su uso, o la falta de planes, programas y proyectos colegiales para ello. Esta alta tendencia en el uso de redes sociales, se pudiera aprovechar más, en el desarrollo del potencial académico de los estudiantes y a su vez incentivar el uso de las tecnologías e-learning, para un mayor rendimiento y cobertura educativa. En la variable uso de la telefonía móvil los resultados se graficaron tal como se observa en la Figura 2. 


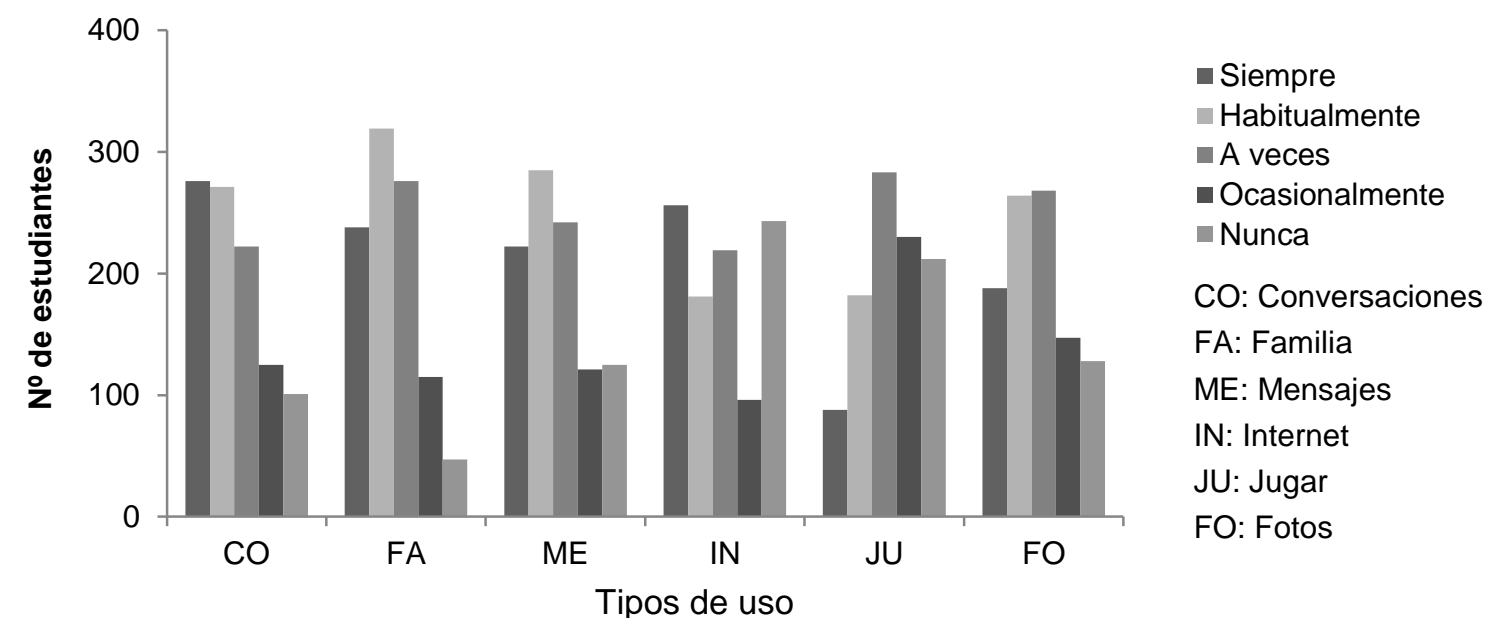

Fig. 2: Frecuencia y uso de móviles de los estudiantes de colegio de 10 y 11

De acuerdo a la escala de calificación, un $27 \%$ de los estudiantes de colegios de 10 y 11 siempre utilizan el teléfono móvil para conversaciones con amigos y un 32\% para conversaciones con la familia, así mismo, un $25 \%$ siempre lo utiliza para conectarse a internet, mientras un $28 \%$ a veces lo utiliza para jugar y un $27 \%$ a veces lo utiliza para hacer fotografías.

Continuando con la tendencia mostrada en el punto anterior, cifras relativamente bajas para el aprovechamiento real de su potencial en cuanto a lo académico, lo que se pudiera explicar por la falta de acceso, dada la ausencia de recursos para adquirir planes de datos, o la conexión a internet o quizás por la tecnología existente (equipos móviles obsoletos para planes de datos), o la falta de planes, programas y proyectos institucionales para su aprovechamiento. Esto muestra tendencia de uso de la telefonía móvil para comunicación verbal (conversación con amigos y familiares), siendo baja hacia el uso del internet y herramientas como fotografía y juegos en un porcentaje menor, dispositivos que podrían aprovecharse aún más para el desarrollo académico de los estudiantes. En la variable uso en el colegio, los resultados se graficaron tal como se observa en la Figura 3.

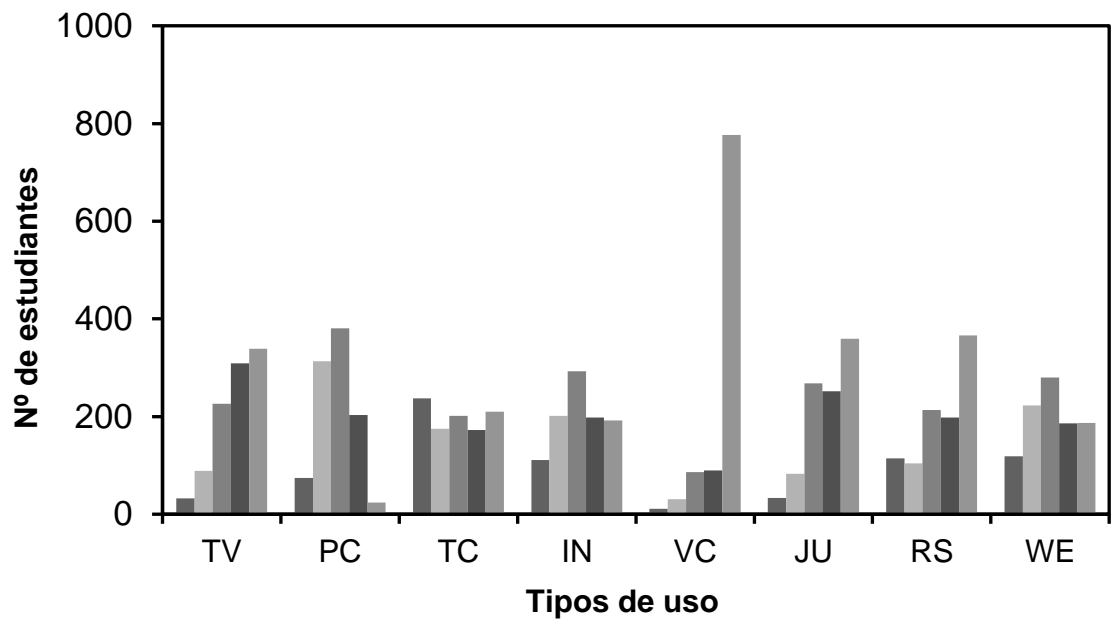

Siempre
Habitualmente
A veces
Ocasionalmente
Nunca
TV: Televisor
PC: Computador
TC: Teléfono Celular
IN: Internet
VC: Videoconsola de juegos
JU: Juegos por computador
RS: Redes sociales
WE: Servicios web educativos

Fig. 3: Frecuencia y uso de TICs de los estudiantes de 10 y 11 en los colegios

De acuerdo a la escala de calificación, un $24 \%$ de los estudiantes de colegios de 10 y 11 siempre utilizan en el colegio el teléfono móvil y en un $12 \%$, redes sociales y servicios web educativos, así mismo, un $31 \%$, habitualmente utiliza el computador y un $20 \%$ internet, mientras un $80 \%$ nunca utiliza video consolas para jugar. Lo que podría explicarse por falta de programas institucionales en los colegios para incentivar el uso de dispositivos móviles para proyectos académicos o los computadores o video consolas para temas relacionados, en parte explicado por la falta de políticas y recursos de asignados por el gobierno nacional, departamental y municipal para el efecto. Esto muestra tendencia de uso de la telefonía móvil para comunicación verbal y de redes sociales, con una baja tendencia hacia el uso de los computadores y del internet, además de usar su tiempo para eventos diferentes a jugar en consolas. Esto indica la posibilidad de aprovechar aún más dichos dispositivos para el desarrollo académico de los estudiantes 
Ahora bien, considerando la gran cantidad de variables utilizadas para medir el uso y apropiación de las TICs para el aprendizaje, es necesario aplicar una técnica estadística que permita realizar una síntesis de la información, reflejada en la reducción de la dimensiones (número de variables) procurando la menor perdida de información posible. Para el efecto se utilizó el software estadístico SPSS y los resultados se muestran en la Tabla 3. Con base en estos resultados (leer porcentaje acumulado de las líneas 1, 2, 3 y 4) se establece lo siguiente: el primer componente explica el 33\% de los resultados, el segundo explica el $61 \%$, el tercero explica el $79 \%$ y el cuarto explica el $89 \%$. Se procede entonces a asignar a cada variable evaluada un componente, de acuerdo al número mayor de los cuatro componentes y como se observa en la Tabla 4. El resultado de dicha asignación se observa en la Tabla 5.

Tabla 3.Componentes principales de las variables

\begin{tabular}{|r|r|r|r|r|}
\hline \multirow{2}{*}{ Componente } & \multicolumn{2}{|c|}{ Autovalores iniciales } & \multicolumn{2}{c|}{ Sumas de las saturaciones al cuadrado } \\
\cline { 2 - 5 } & \% de varianza & \% acumulado & \% de varianza & \% acumulado \\
\hline 1 & 32,82 & 32,82 & 32,82 & 32,82 \\
\hline 2 & 28,75 & 61,56 & 28,75 & 61,57 \\
\hline 3 & 18,33 & 79,9 & 18,33 & 79,91 \\
\hline 4 & 9,82 & 89,73 & 9,82 & 89,73 \\
\hline
\end{tabular}

Tabla 4.Matriz de componentes de las variables para asignación

\begin{tabular}{|l|c|c|c|c|}
\hline \multirow{2}{*}{} & \multicolumn{4}{|c|}{ Componente } \\
\cline { 2 - 5 } & 1 & 2 & 3 & 4 \\
\hline Acom &,- 107 &, 882 &, 409 &, 070 \\
\hline Atem &,- 115 &,- 545 &, 791 &,- 205 \\
\hline Avdj &, 786 &,- 542 &, 085 &,- 225 \\
\hline Fcas &, 676 &,- 163 &, 596 &, 000 \\
\hline Fcol &, 654 &,- 685 &, 021 &, 171 \\
\hline Upcin &, 626 &, 456 &,- 168 &,- 358 \\
\hline Utem &, 640 &, 557 &, 157 &, 427 \\
\hline Hcem &, 175 &, 828 &, 481 &, 081 \\
\hline Hfin &, 722 &, 128 &,- 219 &, 494 \\
\hline Iupc &, 926 &,- 346 &, 002 &, 036 \\
\hline Iuin &, 889 &, 037 &, 224 &,- 273 \\
\hline luvdj &, 520 &, 819 &, 185 &,- 109 \\
\hline Inter &,- 381 &,- 479 &, 356 &, 684 \\
\hline Fac &,- 775 &,- 275 &, 292 &, 135 \\
\hline
\end{tabular}

Tabla 5. Asignación de variables a componentes

\begin{tabular}{|l|l|}
\hline Variable & Representada por el factor \\
\hline Acom & 2: Uso de móvil \\
\hline Aint & 3: Facilidad de acceso a TICs \\
\hline Atem & 1: Acceso a internet \\
\hline Avdj & 1: Acceso a internet \\
\hline Fcas & 1: Acceso a internet \\
\hline Fcol & 1: Acceso a internet \\
\hline Upcin & 1: Acceso a internet \\
\hline Utem & 2: Uso de móvil \\
\hline Hcem & 1: Acceso a internet \\
\hline Hfin & 1: Acceso a internet \\
\hline lupc & 1: Acceso a internet \\
\hline luin & 2: Uso de móvil \\
\hline luvdj & $4:$ Interés por las tecnologías \\
\hline Inter & $4:$ Interés por las tecnologías \\
\hline Fac & 3: Facilidad de acceso a TICs \\
\hline
\end{tabular}


Con estos factores se hace la matriz de correlaciones para corroborar que efectivamente los componentes están bien agrupados. Los resultados se muestran en la Tabla 6. Estos muestran un nivel alto de significancia entre los nuevos 4 factores y ratifica que el acceso uso y de las TICs para el aprendizaje de los jóvenes de último año de educación media en Caldas, se está generando desde el internet, el uso del teléfono móvil, la facilidad y el interés por las tecnologías.

Tabla 6.Matriz de correlación entre factores obtenidos.

\begin{tabular}{|l|l|l|l|l|}
\hline Puntuación de factor 1 para análisis 1 & Sig. (bilateral) & & \multicolumn{2}{|c|}{} \\
\cline { 1 - 3 } Puntuación de factor 2 para análisis 1 & Sig. (bilateral) & 1,000 & & \multicolumn{1}{|}{} \\
\hline Puntuación de factor 3 para análisis 1 & Sig. (bilateral) & 1,000 & 1,000 & \\
\hline Puntuación de factor 4 para análisis 1 & Sig. (bilateral) & 1,000 & 1,000 & 1,000 \\
\hline
\end{tabular}

\section{CONCLUSIONES}

De los resultados encontrados, se pueden obtener las siguientes conclusiones, sobre el acceso y uso de las TICs para el aprendizaje de los jóvenes de último año de educación media en Caldas:

Aunque es muy importante el uso y apropiación de las TICs de los jóvenes preuniversitarios en Caldas, los resultados encontrados muestran que su uso es muy bajo y que no se están aprovechando adecuadamente las mismas en aspectos relacionadas con el aprendizaje, esto conlleva a reflexionar sobre lo que está haciendo el sistema educativo colombiano y del Departamento y sobre las estrategias que pudieran implementarse para un mayor aprovechamiento en el proceso de enseñanza aprendizaje, como sería el desarrollo de planes, programas y proyectos enfocados no solo a una correcta utilización de las mismas, si no a la capacitación para su uso, la implementación de programas de capacitación a los docentes y el desarrollo de plataformas de educación virtual que pudieran apoyar el proceso formativo de los futuros estudiantes universitarios, entre otros.

Los jóvenes de 10 y 11 de los colegios de Caldas, plantean el uso de las TIC's básicamente en lo relacionado con las redes sociales y la comunicación con la familia y amigos, tecnologías que pudieran vincularse más a desarrollos de temáticas educativas que permitan mayores posibilidades de ingreso a una educación universitaria, como serían programas de formación preuniversitaria por parte de los estudiantes de provincia, además de convertirse en una estrategia que podría llevarse a una gran sector de la población, la cual tiene carencia de recursos y oportunidades, que permitan su vínculo a la educación superior.

Uno de los resultados a considerar es que se cuenta con el uso de TIC's, por parte de los jóvenes, pero sigue siendo existente la brecha digital y aunque se viene haciendo esfuerzos importantes por parte del Estado, es fundamental que los Ministerios de educación y tecnologías de información y comunicaciones de Colombia, ejecuten políticas, planes, programas y proyectos que apunten al desarrollo de la formación de docentes y estudiantes conjuntamente, con el apoyo en el acceso y uso de tecnologías para coadyuvar al aprendizaje de estos preuniversitarios.

\section{REFERENCIAS}

ALADI., La Brecha del Conocimiento y la Brecha Digital. In Ponencia presentada en el Simposio sobre la Sociedad de la Información, Córdoba, Argentina (Vol. 24) (2003).

Alcaldia Mayor de Bogota., Ambientes de Aprendizaje para el Desarrollo Humano. Bogota: Fundación Alberto Merani. (2013).

Benavides, C., Tecnología, innovación y empresa. Madrid: Ediciones Piramide. (1998).

Bernete, F., Usos de las TIC, Relaciones sociales y cambios en la socialización de las y los jovenes. CIES PAL/CHAS QUI , Revista Latinoamericana de Comunicación, 97-114 (2009).

Boschma, J., La Generación Einstein. Más listos, más rápidos y más sociables. Comunicar con los jóvenes del siglo XXI. Barcelona: Gestión 2000. (2008). 
Braslavsky, C., Una educación de calidad para todos los jovenes. 47a Conferencia Internacional de Educación de la UNESCO (pág. 155). Ginebra: Oficina Internacional de Educación de la UNESCO. (2004).

Castells, M., De la función de producción agregada a la frontera de posibilidades de producción: productividad, tecnología y crecimiento económico en la era de la información. Barcelona: Real Academica de Ciencias Economicas y Financieras. (2006).

Comunidades Europeas., Competencias Clave para el Aprendizaje Permanente. Belgica: Shutterstock. (2007).

Cuesta, A., y López, M., Brigadas Tecnológicas Rurales. Virtual Educa (pág. n/a). Zaragoza: Virtual Educa. (2008).

Enrique, C., y Alzugaray, G., Modelo de Enseñanza-Aprendizaje para el Estudio de la Cinemática de un Volante Inercial usando Tecnologías de la Información y la Comunicación en un Laboratorio de Física. Formación universitaria, 6(1), 3-12 (2013)..

Espectador. Periódico El espectador. Recuperado el 24 de 03 de 2014, de El espectador: http://www.elespectador.com/noticias/educacion/colombia-vuelve-rajarse-pruebas-de-educacion-pisaarticulo-461894 (01 de 01 de 2000).

García, F., Portillo, J., Romo, J., y Benito, M., Nativos digitales y modelos de aprendizaje. Universidad de País Vasco. (2006).

Giraldo, J., Toro, C., y Jaramillo, F., Aprendiendo sobre la Secuenciación de Trabajos en un Job Shop mediante el Uso de Simulación. Formación Universitaria, 6(4), 27-38 (2013)..

Internet 1, https://drive.google.com/file/d/0B0xZ2BUsIBhfT3RzSGImdVJoZDA/edit?usp=sharing y https://drive.google.com/file/d/0B0xZ2BUsIBhfUDBqc2swYWpTYOk/edit?usp=sharing. (2014)

Lonsdale, D., Embley, D., Ding, Y., Xu, L., y Hepp, M. Reusing ontologies and language components for ontology generation. Data \& Knowledge Engineerin, 318-330 (2010).

López, M., Marulanda, C., y Isaza, G. Cultura organizacional y gestión del cambio y de conocimiento en organizaciones de Caldas. Revista Virtual Universidad Católica del Norte, 117-139 (2011).

Moreno, M., El Desarrollo de Ambientes de Aprendizaje a Distancia. Recuperado el 13 de 11 de 2012, de Maestria en Ingeniería Informática Universidad EAFIT: http://mapas.eafit.edu.co/rid=1JMJSTGFC-1H1Z850GQ/EL_DESARROLLO_DE_AMBIENTES_DE_APRENDIZAJE.pdf. (3 de 3 de 2010).

Muñoz-Cano, J. M., Córdova, J. A., y Priego, H., Dificultades y facilidades para el desarrollo de un proceso de innovación educativa con base en las Tecnologías de la Información y Comunicación (TIC). Formación universitaria, 5(1), 3-12 (2012).

O`Sullivan, K. J., y Azeem, S. W., An Analysis of Collaborative Group Structure Technological Facilitation from a Knowledge Management Perspective. The Electronic Journal of Knowledge Management, 223-230 (2007).

OCDE. Habilidades y competencias del siglo XXI para los aprendices del nuevomilenio en los países de la OCDE. Madrid: Instituto de Tecnologías Educativas. (2010).

Ofsted. ICT in school: Effect of government initiatives, implementation in primary school and effect on literacy. Recuperado el 11 de 3 de 2011, de http://www.ofsted.gov.uk/resources/ict-schools-effect-ofgovernment-initiatives-implementation-primary-schools-and-effect-literacy. (2002).

Osuna, C., y Luna, E., Valores Éticos en la Formación Universitaria de las Áreas de Ciencias Naturales e Ingeniería y Tecnología, en el Contexto de la Sociedad del Conocimiento. Formación universitaria, 4(5), 2936. (2011).

Piscitelli, A., Nativos Digitales. Dieta cognitiva, inteligencia colectiva y arquitecturas de la participación. Buenos Aires: Santillana. (2009). 
Quinche, J. C., y González, F. L., Entornos virtuales 3D, alternativa pedagógica para el fomento del aprendizaje colaborativo y gestión del conocimiento en Uniminuto. Formación universitaria, 4(2), 45-54. (2011).

Tamayo, J., y López, M., Plan Departamental de Ciencia y Tecnologia del Departamento de Caldas. Manizales: Universidad Nacional. (2013).

UNESCO, I. M. Hacia las sociedades del conocimiento. Editorial UNESCO. (2005). 